\title{
A REANALYSIS OF C/O RATIOS IN PLANETARY NEBULAE
}

\author{
C. ROLA \\ DAEC, Observatoire de Paris-Meudon, France \\ Centro de Astrofisica, Universidade do Porto, Portugal \\ and \\ G. STASINSKA \\ DAEC, Observatoire de Paris-Meudon, France
}

It is often mentioned in the literature that, in planetary nebulae,carbon abundancesderived from optical lines are systematically much larger than derived from UV lines. Various explanations have been proposed: line blends, inaccurate atomic physics, temperature fluctuations, non uniform distribution of carbon inside nebulae... Here, we study the effect of random errors in the line intensity measurements.

First, we compiled all the published UV and optical line intensities which allow to estimate $\mathrm{C} / \mathrm{O}$. We then derived the $\mathrm{C} / \mathrm{O}$ ratios by classical empirical methods, assuming that the electron temperature $\mathrm{T}_{\mathrm{e}}$ is given by the [OIII]4363/5007 ratio, and that $\mathrm{C} / \mathrm{O}$ is equal to $\mathrm{C}^{++} / \mathrm{O}^{++}$(abundances derived from CIII]1909/[OIII]5007, CIII]1909/OIII]1661 and CII 4267/[OIII]5007 are denoted by $\mathrm{C}_{\mathrm{uv}} / \mathrm{O}_{\mathrm{opt}}, \mathrm{C}_{\mathrm{uv}} / \mathrm{O}_{\mathrm{uv}}$ and $\mathrm{C}_{\mathrm{opt}} / \mathrm{O}_{\mathrm{opt}}$ respectively). We find that both $\mathrm{C}_{\mathrm{uv}} / \mathrm{O}_{\mathrm{opt}}$ and $\mathrm{C}_{\mathrm{uv}} / \mathrm{O}$ uv are systematically smaller than $\mathrm{C}_{\mathrm{opt}} / \mathrm{O}_{\mathrm{opt}}$.

In order to understand to what extent observing conditions alone affect observational diagrams, we have performed the following numerical experiment. We have randomly selected a sample of 100 objects out of a population of fictive planetary nebulae having $\mathrm{C} / \mathrm{O}$ distributed around 0.8 , with a dispersion of 0.3 dex. Temperatures were assumed uniform, but varying from object to object, with a mean of $12000 \mathrm{~K}$ and a dispersion of $1400 \mathrm{~K}$. The observational errors in the line intensities were simulated by randomly selecting the intensities out of a Gaussian distribution centered on the true intensity, with a dispersion chosen so as to reproduce the typical uncertainties stated in observational papers. A line was considered to be detected when its intensity was at least 1.5 times greater than the noise. To these simulated intensities, we have applied the same empirical methods as above to derive the $\mathrm{C} / \mathrm{O}$ ratios.

The simulated diagrams comparing $\mathrm{C}_{\mathrm{uv}} / \mathrm{O}_{\mathrm{opt}}, \mathrm{C}_{\mathrm{uv}} / \mathrm{O}_{\mathrm{uv}}$ and $\mathrm{C}_{\mathrm{opt}} / \mathrm{O}_{\mathrm{opt}}$ show qualitatively the same patterns as the observational ones. There is no systematic bias in $\mathrm{C} / \mathrm{O}$ when derived from $\mathrm{C}_{\mathrm{uv}} / \mathrm{O}_{\mathrm{opt}}$ because these lines are very strong. On the contrary, $\mathrm{C}_{\mathrm{opt}} / \mathrm{O}_{\mathrm{opt}}$ tends to overestimate the true $\mathrm{C} / \mathrm{O}$, especially at small $\mathrm{C} / \mathrm{O}$ ratios. Indeed, there is a selection effect against nebulae in which random realizations of the CII 4267 intensities are below the true value, because these weak lines are often lost in the noise.

We conclude that random uncertainties in the line intensity measurements are an important factor in explaining the discrepancy between UV and optical determinations of $\mathrm{C} / \mathrm{O}$ ratios.

It is unwise to combine $\mathrm{C}_{\mathrm{uv}} / \mathrm{O}_{\mathrm{opt}}$ with $\mathrm{C}_{\mathrm{opt}} / \mathrm{O}_{\mathrm{opt}}$ for statistical studies of planetary nebulae, because the systematic differences depend on the signal-to-noise of the observations. 